\title{
An Early Broad Competence of Motoneurons to Express ER81 Is Later Sculpted by the Periphery
}

\author{
Guoying Wang and Sheryl A. Scott \\ Department of Neurobiology and Anatomy, University of Utah School of Medicine, Salt Lake City, Utah 84132
}

The ETS transcription factor ER81 is expressed in sensory neurons and motoneurons that innervate the adductor and femorotibialis muscles in chick hindlimb and is essential for the development of monosynaptic connections between these two populations of neurons. Neurons need a signal(s) from limb bud mesoderm to initiate ER81 expression. It is not known whether the mature expression pattern arises because adductor and femorotibialis motoneurons are uniquely competent to respond to peripheral signals and express ER81, or whether all motoneurons are competent to express ER81, but normally only adductor and femorotibialis motoneurons are exposed to the requisite activating signal. To investigate these possibilities, we examined $E R 81$ expression in motoneurons that encountered limb tissue surgically mismatched with their target identity at stages after motor pool identities are established. We found that $E R 81$ expression was not invariably linked to motor pool identity or target innervation and was more malleable in later-born femorotibialis motoneurons than in earlier-born adductor motoneurons. We also found that $E R 81$ expression is regulated differently in sensory neurons and motoneurons. Most striking was the observation that motoneurons caudal to the normal adductor and femorotibialis pools could express ER81 when exposed to the appropriate peripheral signals, although this competence did not extend through the entire lumbosacral (LS) region. Thus, it appears that a prepattern of competence to express ER81 is established in early LS motoneurons, most likely in concert with their target identity, and that the expression domains of motoneurons are subsequently refined by peripheral signals at later stages.

Key words: hindlimb; ETS transcription factors; chick; ER81; motor neuron; sensory neurons; dorsal root ganglia; development

\section{Introduction}

Motoneurons that innervate individual muscles in limb are organized into pools that occupy characteristic positions in the lateral motor column (LMC) of the spinal cord (for review, see Landmesser, 2001). Each motoneuron in the pool receives monosynaptic connections from sensory neurons innervating the same muscle (Wenner and Frank, 1995). Although the molecular mechanisms responsible for the individuation of motor pools are unknown, each pool ultimately acquires a unique molecular signature that is important for its further development (Price et al., 2002). For example, at lumbosacral (LS) levels in chick, the ETS transcription factor ER81 is expressed in motor and sensory neurons that innervate the adductor (Add) and external femorotibialis (Fem) muscles soon after their axons grow into the limb (Lin et al., 1998). ER81 is required for development of monosynaptic connectivity in ventral horn (Arber et al., 2000) and may regulate selective recognition between Add and Fem sensory neurons and motoneurons (Price et al., 2002).

Little is known about the molecular mechanisms that control

Received June 10, 2004; revised Sept. 16, 2004; accepted Sept. 18, 2004.

This work was supported by National Institutes of Health Grant NS 16067 to S.A.S. We thank Drs. Richard Dorsky and Maureen Condic for insightful comments on a previous version of this manuscript and members of the Condic laboratory for lively discussions throughout the course of this research. We also thank Dr. Silvia Arber for supplying the ER81 plasmid, Dr. Frances Lefcort for trkC antibody, and Dr. Alan Light for allowing us to use his electrophysiology equipment and generously assisting us with these experiments.

Correspondence should be addressed to Sheryl A. Scott, Department of Neurobiology and Anatomy, University of Utah School of Medicine, 20 North 1900 East, Salt Lake City, UT 84132. E-mail: sheryl@neuro.utah.edu.

DOI:10.1523/JNEUROSCI.3409-04.2004

Copyright $\odot 2004$ Society for Neuroscience $\quad$ 0270-6474/04/249789-10\$15.00/0
ER81 expression. ER81 protein first appears about the time that axons grow into limb muscles. However, neurons need an earlier signal from limb to initiate expression, because ER81 expression is markedly decreased by limb bud ablation (Lin et al., 1998). Sensory neurons require muscle-derived neurotrophin 3 (NT3) (Patel et al., 2003), but the requisite peripheral signal(s) for motoneurons is unknown.

We can envision two scenarios by which the periphery could control ER81 expression in unique motor pools. First, competence to express ER81 may be established in selected motoneurons early in development in concert with their target identity (Matise and Lance-Jones, 1996). Signals from the limb would simply activate expression specifically in these neurons. Alternatively, any motoneuron could potentially express ER81, but only outgrowing Add and Fem motor axons normally encounter ER81-inducing signals in the limb. Thus, establishment of motor pool identity may restrict ER81 expression to Add and Fem neurons simply by determining the initial trajectory and ultimate target muscle of the neurons (cf. Lin et al., 1998).

Previous studies did not distinguish between these possibilities, because they did not challenge motoneurons with inappropriate peripheral signals (Lin et al., 1998). Here we examined ER81 expression in motoneurons that encountered limb tissue mismatched with their target identity at stages after motor pool identities were established (Matise and Lance-Jones, 1996). We found that ER81 expression was not invariably linked to either motor pool identity or target innervation. Importantly, motoneurons caudal to the normal Add and Fem pools could express 
ER81 when exposed to the appropriate peripheral signals, although this competence did not extend through the entire LS region. Thus, it appears that early patterning events in the neural tube (Ensini et al., 1998; Jessell, 2000; Dasen et al., 2003; Omelchenko and Lance-Jones, 2003) establish competence domains for ER81 expression in LS motoneurons. Subsequently, additional peripheral signals activate ER81 expression exclusively in restricted populations of competent motoneurons, the Add and Fem motor pools. Some of this work has been published previously in abstract form (Wang and Scott, 2003).

\section{Materials and Methods}

\section{General}

Fertile white Leghorn chick eggs from a local supplier were incubated in a humidified forced-draft incubator at $38^{\circ} \mathrm{C}$. Embryos were staged according to Hamburger and Hamilton (1951) at the time of surgery and at the time they were killed.

\section{Surgical manipulations}

Several types of surgical manipulations were performed on embryos between stage (St.) 15 and 18 [embryonic day 2.5 (E2.5) to E3]. A window was cut in the shell over the embryo. The vitelline and amniotic membranes over the embryo were opened, and the area was stained lightly with $0.5 \%$ neutral red in sterile Ringer's solution (Scott, 1984). The desired portion of the tissue was excised with sharpened tungsten needles. Eggs were sealed with paraffin and a coverslip and returned to the incubator until the desired stages. In each operated embryo, except those with neural tube transplantations, we subsequently compared ER81 expression pattern and/or peripheral connectivity with that observed on the control side.

Limb bud removal. To confirm the stages at which peripheral signals are required to initiate ER81 expression (Lin et al., 1998), hindlimb buds or prospective hindlimb tissue was removed unilaterally between St. 15 and 17. Embryos were allowed to survive until St. 28-29 (E5.5-E6.5) when ER81 expression was examined.

\section{Mismatching motoneurons and limb}

To create a mismatch in the segmental level of motoneurons and adjacent limb and/or somites, three types of operations were performed:

Limb shift. One limb bud was shifted forward by one to three segments at St. 17, before significant sensory and motor axon outgrowth, as described previously (Lance-Jones and Landmesser, 1981; Wang and Scott, 1999, 2002). In addition, in five sham-operated embryos, one limb bud was simply removed and replaced.

Anteroposterior limb reversal. A hindlimb was reversed $180^{\circ}$ along the anteroposterior (A-P) axis, leaving the dorsoventral axis unaltered in St. 17-18 embryos, as described by Landmesser and O’Donovan (1984). Briefly, the right limb bud of one embryo was transplanted to the left side of a second embryo, whose left limb had previously been removed, and vice versa.

Neural tube transplantation. Several segments of neural tube were heterotopically transplanted between different rostrocaudal LS levels at St. 16.

(1) Caudal-to-rostral transplantation. In some embryos, the host neural tube was removed from the last thoracic (T) segment (T7; opposite somite 26) through segment LS3 (opposite somite 29) or from segments LS1-LS3 and replaced with three to four segments of more caudal neural tube from donor embryos of the same stage. Progressively more caudal donor neural tube regions were transplanted into different host embryos, with the most rostral transplants beginning at segment LS3.

(2) Rostral-to-caudal transplantation. In other embryos, the host neural tube was removed from segments LS3 to LS5 or LS6 and replaced with rostral donor neural tube, beginning with segment T7 or LS1 and extending three to four segments caudally. Because somites had not formed throughout the entire LS regions at the time of surgery, it was sometimes difficult to determine the exact number of segments being removed at caudal levels. In each case, we tried to match the length of the transplant and excised tissue.

\section{In situ hybridization}

ER81 expression was examined in embryos from St. 28, $\sim 1 \mathrm{~d}$ after the initiation of expression in motoneurons (Lin et al., 1998) and before extensive cell death (Calderó et al., 1998), through St. 35 (E9). Embryos were fixed in $4 \%$ paraformaldehyde overnight at $4^{\circ} \mathrm{C}$ and then rinsed several times in $0.1 \mathrm{~m}$ PBS. The pattern of ER81 expression was determined by whole-mount or section in situ hybridization using a digoxigenin-labeled RNA antisense probe (a Bluescript plasmid with an ER81 insert was kindly provided by Dr. Silvia Arber, University of Basel, Basel, Switzerland). The methods used for section in situ hybridization were modified from Birren et al. (1993). The same process was used for whole-mount in situs, but the time for each step was longer.

\section{Immunohistochemistry}

Tyrosine receptor kinase (trk)C and ER81 were examined in adjacent cryosections of thoracic dorsal root ganglia (DRG) in four embryos (St. 30-32) with rostral limb shifts. Every other section was reacted for ER81. The remaining sections were stained with anti-trkC (kindly provided by Dr. Frances B. Lefcort, Montana State University, Bozeman, MT) amplified with tyramide signal amplification (New England Nuclear, Boston, MA), as described by Wang and Scott (2000). Darkly labeled ER81positive and trkC-positive neurons were counted in DRGs T6 and T7 on control and experimental sides of each embryo.

\section{Analysis of motoneuron connectivity patterns}

Electrophysiology. Peripheral connectivity of motoneurons was mapped electrophysiologically at St. 32-35 (E7.5-E9) in six embryos with rostral limb shifts and five embryos with A-P limb reversals. Embryos were placed in a bath of oxygenated Ringer's solution at room temperature and were decapitated, eviscerated, and a ventral laminectomy was performed. The hindlimbs were skinned and the muscles freed of connective tissue. Motoneurons at different segmental levels were activated by stimulating the ventral surface of the spinal cord opposite each spinal nerve with a suction electrode, as described by Lance-Jones and Landmesser (1980). Electromyograms (e.m.g.s) were recorded from individual muscles in the thigh of both control and operated limbs with a small concentric electrode.

Embryos were subsequently fixed and sectioned for ER81 in situ hybridization. To compare expression and connectivity in the same embryos, we plotted the regions of spinal cord at which stimulation activated the adductor or femorotibialis muscle and the distribution of ER81-positive motoneurons in the medial compartment of the lateral motor column (MLMC) or lateral compartment of the LMC (LLMC), respectively, on the same axis (see Figs. 2, 5). To construct these figures, spinal cord segments were normalized to an arbitrary standard length, with the division between segments defined as the point midway between adjacent DRGs. Efficacious stimulation opposite a spinal nerve is represented as occupying the entire segment; the proportion of sections through a spinal segment that showed ER81 expression is represented as a fraction of the standard segment length.

Retrograde labeling. Individual muscles were injected with 10\% HRP in three embryos with A-P limb reversals and three embryos with neural tube transplants, as described by Matise and Lance-Jones (1996). After incubation at $30^{\circ} \mathrm{C}$ for $6 \mathrm{hr}$, embryos were fixed in $4 \%$ paraformaldehyde and cryosectioned. Every other section was reacted with diaminobenzidine, and the remaining sections were examined for ER81 expression. In addition, 1,1'-dioctadecyl-3,3,3',3-tetramethylindocarbocyanine perchlorate (diI; Molecular Probes, Eugene, OR) $(2.5 \mathrm{mg} / \mathrm{ml}$ dimethylformamide) was injected into individual muscles in four embryos with A-P limb reversals. After incubation at $29^{\circ} \mathrm{C}$ for $6 \mathrm{hr}$, embryos were fixed in $4 \%$ paraformaldehyde at $37^{\circ} \mathrm{C}$ for 1 week and viewed as whole mounts.

\section{Results}

The ETS transcription factor ER81 is essential for the development of an important neural circuit, monosynaptic connections responsible for the stretch reflex (Arber et al., 2000). In chick, ER81 is expressed in Add motoneurons in the MLMC in spinal cord segments LS1-LS2 and in external Fem motoneurons in the LLMC in segments LS2-LS3 (Fig. 1, left). ER81-positive sensory 




Figure 1. ER81 expression is reduced in femorotibialis motoneurons after rostral limb shifts. Left, Top, Ventral view of the spinal cord and DRGs from thoracic segment T7 through LS segment LS4 of a sham-operated embryo [St. 31 (E7.5)] in which one limb (represented on the right) was removed and replaced at St. 17. Left, Bottom, Ventral view of the spinal cord and DRGs from a St. 30 (E7) embryo in which one limb (represented on the right) was shifted two to three segments rostrally at St. 17. Right, Sections through the ventral spinal cord of another limb-shifted embryo, St. 31 (E7.5). In both embryos with shifted limbs, ER81 expression in MLMC (M) motoneurons that innervated the shifted limb is nearly identical to that of control adductor motoneurons (A). In contrast, very few LLMC ( $L$; arrow) motoneurons expressed ER81 after limb shifts, although the lateral motor column (encircled by white dots in panels LS2 and LS3) is quite large. Note that ER81 is nearly identical in both motor pools after sham operations. $\mathrm{F}$, Femorotibialis motoneurons.

neurons are located in the ventrolateral region of DRGs, a distribution characteristic of proprioceptive neurons (see Fig. 3). Initiation of ER81 expression requires signals from the periphery, because limb bud ablation almost completely eliminates expression (Lin et al., 1998). It is not known whether all motoneurons are competent to respond to this peripheral signal(s), but only Add and Fem neurons normally encounter the signals. Alternatively, competence to respond to peripheral signals and express ER81 may be a unique property of Add and Fem neurons, established early in development in concert with their target identity. To investigate the role of peripheral signals in regulating ER81 expression, we examined expression patterns in motoneurons and sensory neurons that entered the limb from novel positions along the A-P axis. All of our manipulations were performed at stages after the target identity of motoneurons had been established (Matise and Lance-Jones, 1996).

\section{ER81 expression after limb shift}

As a first approach, we shifted limb buds one to three segments rostrally at St. 17 (E2.5) and examined ER81 expression with in situ hybridization at St. 28-32. In previous studies, motor and sensory innervation patterns were affected differently by limb shifts. Whereas motoneurons tended to innervate their embryologically correct muscles after limb shifts (Lance-Jones and Landmesser, 1981; Wang and Scott, 1999), sensory innervation of muscle was more widespread than normal and was generally from more rostral segments than normal (Wang and Scott, 1999). Thus, we anticipated that limb shifts might influence ER81 expression differently in motor and sensory neurons.

Limb shifts had little effect on ER81 expression in MLMC motoneurons but greatly reduced expression in the LLMC. As illustrated in Figure 1, ER81-positive MLMC motoneurons were located in the same spinal segments as control Add motoneurons in all embryos examined $(n=21)$. Moreover, the size of the ER81-positive MLMC pool was similar on both sides in most embryos. In contrast, although the LLMC itself was quite large, very few LLMC motoneurons expressed ER81 after limb shifts. The labeled cells were, however, located in the segments appropriate for Fem motoneurons. There were no obvious differences in the location or numbers of ER81-positive motoneurons in sham-operated embryos in which one limb bud was simply removed and replaced $(n=5)$ (Fig. 1$)$.

To determine whether peripheral innervation patterns could account for the differences in ER81 expression in MLMC and LLMC motor pools, we compared motoneuron connectivity patterns mapped electrophysiologically and ER81 expression in embryos with limb shifts, as described in Materials and Methods. This approach allowed us to assess motor innervation of several different muscles (both appropriate and inappropriate) in each embryo in which we subsequently examined ER81 expression. Activation of the Add and Fem muscles in shifted limbs generally required a more intense stimulus than activation of control muscles, suggesting that fewer motoneurons innervated these muscles. Consistent with ER81 expression, Add motoneurons appeared to innervate their appropriate muscle more accurately and more robustly than Fem motoneurons. Stimulation of motoneurons in segments LS1-LS2 always activated the Add muscle (Fig. 2, top), and motoneurons in LS1 rarely innervated inappropriate postaxial muscles (data not shown). Activation of the Fem muscle was less reliable, and we occasionally failed to detect innervation of the Fem from segment LS3 (Fig. 2, bottom) [see also Wang and Scott (1999), their Fig. 7]. It is possible, however, that a few motoneurons in segment LS3, and even more caudal segments, innervated the Fem but escaped detection, because we only sampled e.m.g.s at a single position in this large muscle and did not distinguish between the external and internal heads. In contrast, motoneurons in segments LS2 and LS3 always innervated several postaxial muscles that are normally served by motoneurons in more caudal segments (data not shown). Innervation of inappropriate muscles clearly supported the survival of motoneurons in segments LS2 and LS3 but did not appear to activate expression of ER81. Thus, the reduced expression of ER81 in LLMC most likely reflects the failure of many Fem motoneurons to innervate the Fem muscle.

Normally, a few scattered cells in ventral thoracic spinal cord expressed ER81. After limb shifts, thoracic motoneurons often innervated the Add and/or Fem muscles. Despite this, there was no increase in ER81 expression in thoracic motoneurons, suggesting that peripheral signals from target muscles may not be sufficient to activate ER81 expression in all motoneurons. The failure of thoracic motoneurons to express ER81 is consistent with the observation that thoracic motoneurons do not express the ETS transcription factor Pea3 in response to glial cell linederived neurotrophic factor (GDNF), a muscle-derived factor that induces Pea-3 expression in cervical LMC motoneurons (Haase et al., 2002).

Whereas ER81 is selectively expressed in Add and Fem motoneurons from its earliest appearance during normal develop- 

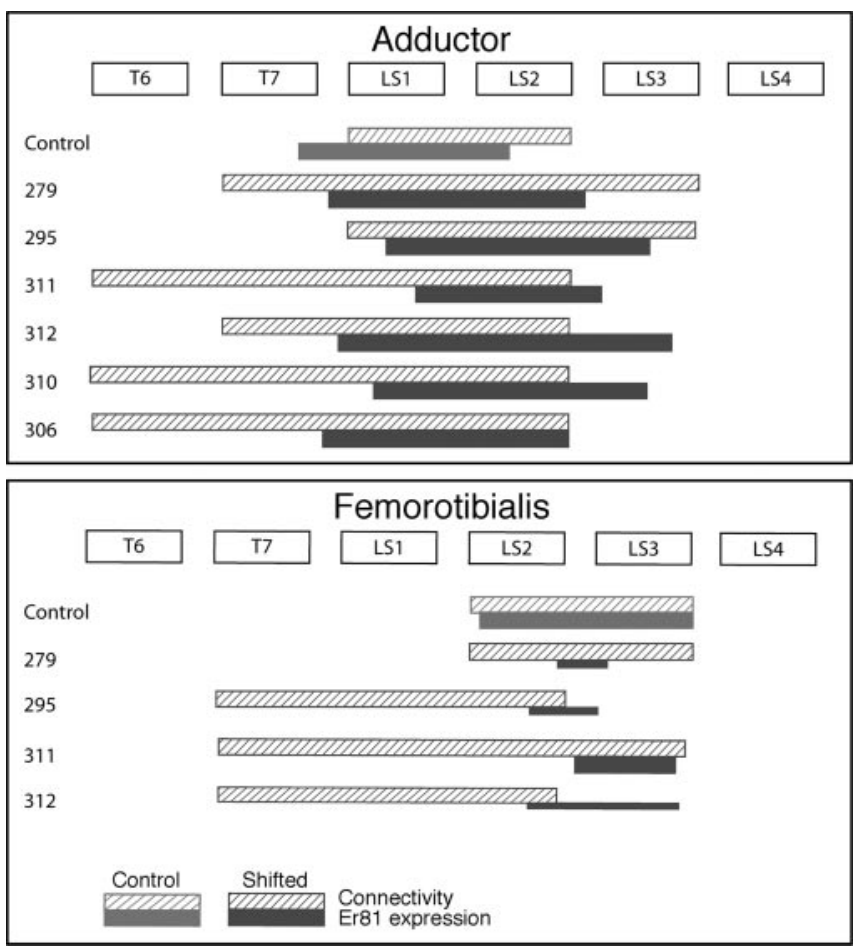

Figure 2. Comparison of motoneuron connectivity and ER81 expression in embryos with rostral limb shifts. Each pair of bars represents a single embryo, designated by the number on the left. A single representative control embryo (gray; top bars in each panel) is shown for comparison. Hatched bars represent the segmental levels at which stimulating the ventral spinal cord elicited e.m.g.s from the adductor and femorotibialis muscles (top and bottom panels, respectively). Solid bars represent the distribution of ER81-positive neurons in MLMC (top panel) and LLMC (bottom panel) motoneurons. The thinner solid bars in the bottom panel indicate that fewer than normal LLMC motoneurons expressed ER81. Note that MLMC and LLMC motoneurons in segments LS1-LS3, the normal location of adductor and femorotibialis motor pools, express ER81 when they innervate the adductor and femorotibialis muscles, respectively. In contrast, motoneurons in thoracic segments do not express ER81, even when they innervate these muscles. Activation of muscles in shifted limbs generally required a more intense stimulus than activation of control muscles, as if fewer motoneurons than normal innervated these muscles, which most likely accounts for the reduced expression of ER81 in LLMC motoneurons.

ment, both Pea3 and ER81 are initially widely coexpressed in DRG neurons. Expression becomes restricted to Add and Fem sensory neurons about the time that monosynaptic connections are established (Lin et al., 1998). At the stages of embryos we studied, ER81 expression was widespread in sensory neurons, and labeled neurons were present in all lumbosacral DRGs. Rostral limb shifts dramatically altered this distribution. The pattern of ER81 expression in sensory neurons was shifted several segments rostrally, consistent with previously reported shifts in sensory innervation patterns (Wang and Scott, 1999, 2002). Some neurons in thoracic DRGs T5-T7 expressed ER81, whereas neurons in DRGs LS7 and LS8 did not (Fig. 3). Moreover, in some DRGs that innervated shifted limbs, the labeled neurons were more broadly distributed throughout the ganglion rather than being restricted to a ventrolateral rim of cells.

In lumbosacral DRGs in control embryos, most trkC-positive neurons, which are primarily proprioceptive, also express an ETS transcription factor (Lin et al., 1998). In contrast, few trkC+ thoracic DRG neurons expressed either ER81 (Table 1) or Pea3 (data not shown). Limb shifts increased both the number of trkC+ and ER81+ neurons in thoracic DRGs, such that the percentage of trkC+ neurons that also expressed ER81 (Table 1) approached that of lumbosacral DRGs (Lin et al., 1998). For



Figure 3. ER81 is expressed in more rostral DRGs than normal after limb shifts. Images are through the approximate midsection of representative DRGs of a single St. 31 (E7.5) embryo with one limb shifted rostrally; lateral is toward the outer edge of each panel. Note that the pattern of ER81 expression is shifted rostrally in DRGs serving the operated limb, and in some DRGs the labeled cells are more widely scattered than normal. 
Table 1. Comparison of TrkC ${ }^{+}$and $E R 81^{+}$expression in thoracic DRG neurons in embryos with rostral limb shifts

\begin{tabular}{|c|c|c|c|c|c|c|c|c|}
\hline \multirow[b]{3}{*}{ Ganglion } & \multicolumn{3}{|l|}{ Control } & \multicolumn{3}{|l|}{ Shifted } & \multirow{2}{*}{\multicolumn{2}{|c|}{ Shifted/Control }} \\
\hline & & & Percentage & & & Percentage & & \\
\hline & $\mathrm{TrkC}^{+}$ & $E R 81^{+}$ & $\mathrm{ER} 81^{+} / \mathrm{TrkC}^{+}$ & TrkC $^{+}$ & $E R 81^{+}$ & $\mathrm{ER} 81^{+} / \mathrm{TrkC}^{+}$ & $\mathrm{TrkC}^{+}$ & $E R 81^{+}$ \\
\hline T6 & $120.8 \pm 21.0$ & $24.5 \pm 3.0$ & $20.6 \pm 3.1$ & $188.0 \pm 58.4$ & $105.5 \pm 71.9$ & $53.6 \pm 27.2$ & $1.6 \pm 0.5$ & $4.4 \pm 2.9$ \\
\hline $\mathrm{T} 7$ & $128.0 \pm 47.9$ & $37.8 \pm 15.2$ & $31.8 \pm 13.2$ & $261.5 \pm 104.6$ & $200.5 \pm 112.1$ & $73.2 \pm 12.8$ & $2.2 \pm 0.9$ & $6.0 \pm 3.8$ \\
\hline
\end{tabular}

$\mathrm{TrkC}^{+}$and darkly labeled $E R 81^{+}$neurons were counted in adjacent sections through thoracic DRGs T6 and T7 in four embryos (St. 30 -32) in which one limb bud was shifted rostrally at St. 17. Values are mean \pm SD. The large variance in number of labeled neurons on the shifted side is attributable to differences in the age of the embryos as well as differences in the extent of limb shift.

example, in control thoracic DRG T7, 30\% of trkC+ neurons expressed ER81, whereas after limb shifts, nearly 75\% were $E R 81+$. The most likely explanation for the altered patterns of expression of trkC and ER81 is that after limb shifts, proprioceptive sensory neurons that innervate the shifted limb are rescued from naturally occurring cell death (Hamburger and LeviMontalcini, 1949). Subsequently, innervation of limb muscles activates expression of ER81 in these rescued neurons, as well as in many of the normal trkC+ thoracic neurons, leading to the large increase in the percentage of $E R 81+/ \operatorname{trkC}+$ neurons.

\section{ER81 expression after A-P limb reversal}

Limb shifts create only a small disparity between the segmental level of motoneurons and the limb, which might account for the nearly normal pattern of ER81 expression in MLMC motoneurons. To create a greater disparity, we reversed limb buds around the A-P axis leaving the dorsoventral axis intact, as described in Materials and Methods. Such reversals exposed nascent motoneurons in segments LS1-LS3 to peripheral signals from posterior limb mesoderm. Despite this, MLMC and LLMC motoneurons expressed ER81 in all embryos $(n=19)$. However, the ER81 pattern differed from controls in a consistent and intriguing manner; expression in the LLMC pool was shifted caudally, extending one to two segments more caudal than normal in all operated embryos, often reaching far into segment LS5. The MLMC pool also extended up to one segment caudally in twothirds of the embryos (Figs. 4, 5) but was less severely affected than the LLMC pool. Thus, A-P limb reversals revealed an unexpected competence to express ER81 in motoneurons outside the normal Add and Fem domains of expression.

Furthermore, physiological assessments indicated that expression of ER81 was not invariably coupled to peripheral connectivity. For example, ER81 was expressed in MLMC motoneurons in segments LS1 and LS2, although motoneurons in these segments seldom innervated the Add muscle (Fig. 5) but instead innervated muscles in posterior limb, such as the caudilioflexorius (data not shown). Conversely, MLMC motoneurons caudal to LS4 often innervated the Add but never expressed ER81, suggesting the ER81 pattern in MLMC motoneurons was essentially established at the time of surgery.

The relationship between ER81 and connectivity was also complex for LLMC motoneurons and muscle (Fig. 5). Stimulation of the spinal cord indicated that after A-P limb reversal, motoneurons in segment LS2 innervated inappropriate muscles in posterior limb (cf. Landmesser and O'Donovan, 1984) (data not shown) and generally did not innervate the Fem muscle. Instead, the Fem muscle in reversed limbs was innervated by motoneurons in segments LS3 through LS6-LS8 (Figs. 5, 6). ER81 was expressed in LLMC motoneurons only in the more rostral segments (LS3-LS5), but never in more caudal segments (LS6-LS8), even when motoneurons in these segments innervated the Fem muscle. HRP injections into the Fem muscle in three embryos confirmed that LLMC motoneurons in LS3-4 or
LS5 that innervated the Fem muscle expressed ER81, whereas those in more caudal segments did not (Fig. 6A). DiI injections into the Fem muscle $(n=4)$ (Fig. $6 B)$ in A-P-reversed limbs further verified that the Fem was innervated by motoneurons caudal to those that expressed ER81 in other embryos with limb reversals. Thus, ER81 expression in LLMC motoneurons was highly correlated with innervation of the Fem muscle, suggesting that the Fem may provide an important signal for activating ER81 expression. However, innervation of the Fem muscle is clearly not sufficient, because it does not induce ER81 expression in the most caudal lumbosacral motoneurons.

As in control embryos, ER81 was widely expressed in DRGs along the rostrocaudal axis after A-P limb reversals at the stages studied. Although the relative numbers of labeled neurons varied somewhat between individual control and experimental DRGs in about one-half of operated embryos, there were no obvious, consistent differences in expression. The finding that the ER81 ex-

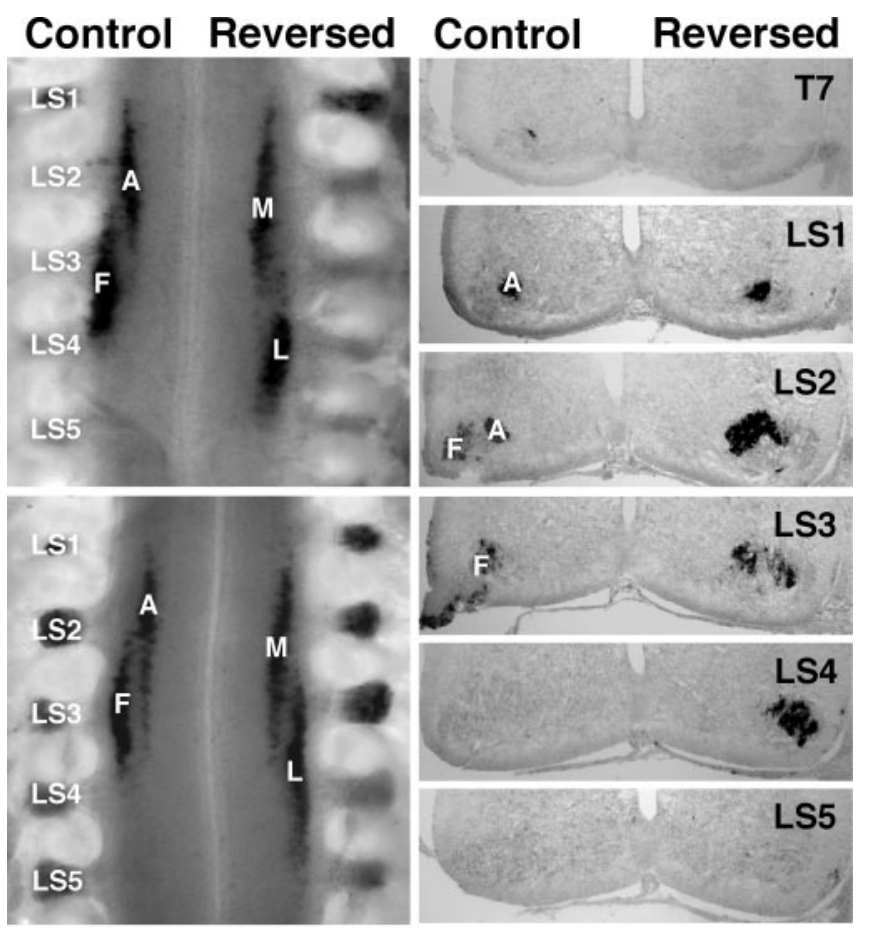

Figure 4. A-P limb reversals reveal a broad competence of MLMC and LLMC neurons to express ER81. Left, Ventral view of the spinal cord and DRGs from segments LS1-LS5 of two St. 31 (E7.5) embryos in which one limb (represented on the right) was reversed $180^{\circ}$ along the A-P axis at St. 17, leaving the dorsoventral axis intact. Right, Sections through the ventral spinal cord of a St. 33 (E8) embryo with an A-P reversed limb. The control LS3 spinal cord was distorted by the suction electrode used to stimulate motoneurons. In all three embryos with A-P-reversed limbs, ER81 expression in MLMC (M) motoneurons that innervated the reversed limb extended up to one segment more caudal than the normal adductor pool (A). More strikingly, ER81 expression in LLMC (L) motoneurons that innervated the reversed limb extended as much as two segments more caudal than the normal femorotibialis pool (F). 
pression pattern is not clearly reversed in DRGs after A-P limb reversals suggests that the initial expression of ER81 in sensory neurons can be activated by innervation of any limb muscle. Whether refinement to the final mature pattern of expression in DRGs is influenced by innervation of inappropriate muscles remains to be determined.

Heterotopic neural tube transplantation After A-P limb reversal, ER81 expression in the LLMC extended one to two segments more caudally but never past LS5, although nascent motoneurons in these segments lay adjacent to mesoderm derived from segments LS1-LS3 and eventually innervated the Fem muscle. However, in these embryos, the tissue immediately adjacent to the spinal cord, the paraxial mesoderm or somites, was not manipulated and could have provided cues that balanced or counteracted those derived from limb mesoderm. To test whether concordant signals from both anterior somitic and limb mesoderm would induce caudal LLMC neurons to express ER81, we transplanted progressively more caudal regions of neural tube into segmental levels LS1-LS3 at St. 16, a stage at which motor pool identities are definitively established (Matise and Lance-Jones, 1996). Despite the relative maturity of transplanted neural tube, motoneurons derived from segments LS4 and LS5, which normally never express ER81, expressed ER81 when transplanted rostrally. Moreover, expression was always restricted to the LLMC, characteristic of Fem motoneurons, even when neural tube was transplanted into segments LS1 or LS2, where ER81 is normally expressed in MLMC (Fig. 7A). In contrast, motoneurons from more caudal segments did not express ER81, even when HRP injections demonstrated that these transplanted motoneurons innervated the Fem muscle (data not shown). This dramatic, but restricted, ability of caudal LLMC motoneurons to express ER81 after rostral transplantation is similar to the patterns observed after limb reversals.

Motoneurons derived from segments LS1-LS3 expressed ER81 when neural tube segments were transplanted caudally. ER81 was expressed primarily in MLMC neurons, characteristic of the Add pool in these embryos (Fig. 7B).

These results, together with those of limb reversals, suggest that competence domains for ER81 expression are established in both the MLMC and LLMC before axon outgrowth. In the LLMC, and to a lesser extent the MLMC, the competence domain extends well beyond the normal Add and Fem motor pools, because ER81 expression can be induced in motoneurons that normally never express this transcription factor at stages after motor pool identities are established. Previous studies involving neural tube rotations did not reveal this broad competence and plasticity of ER81 expression in relatively mature motoneurons (Lin et al., 1998), most likely because caudal LS motoneurons in previous studies were not exposed to limb-derived ER81-activating signals. However, competence to express ER81 extends only through segment LS5. More caudal motoneurons, when exposed to signals that activate ER81 expression at rostral levels, do not respond to these signals, at least at the stages studied. This broad competence to express ER81 in response to limb-derived signals was unexpected, because GDNF, an early limb-derived regulator of Pea3, activates Pea3 expression only in the appropriate motoneurons (Haase et al., 2002). It will be of interest to determine whether LLMC motoneurons lose the competence to respond to ER81-inducing signals at later stages.

\section{Adductor versus femorotibialis motoneurons}

Previous studies have shown that expression of ER81 requires a signal from limb mesoderm at a stage before axons have grown out to peripheral target muscles (Lin et al., 1998). As described above, all of our experimental manipulations created a mismatch between the A-P identity of limb mesoderm and the segmental level of motoneurons, ultimately causing some motoneurons to innervate inappropriate target muscles. Exposure of motoneurons to novel mesoderm and innervation of inappropriate muscles had minor effects on ER81 expression in MLMC motoneurons but dramatically altered expression in LLMC motoneurons. One possible explanation for this difference is that motoneurons in the MLMC are born before (and therefore are more mature) than motoneurons in the LLMC. Thus, many Add motoneurons may have already encountered sufficient signals from the appropriate mesoderm to activate and maintain expression of ER81 by the time that we experimentally mismatched the neurons with 


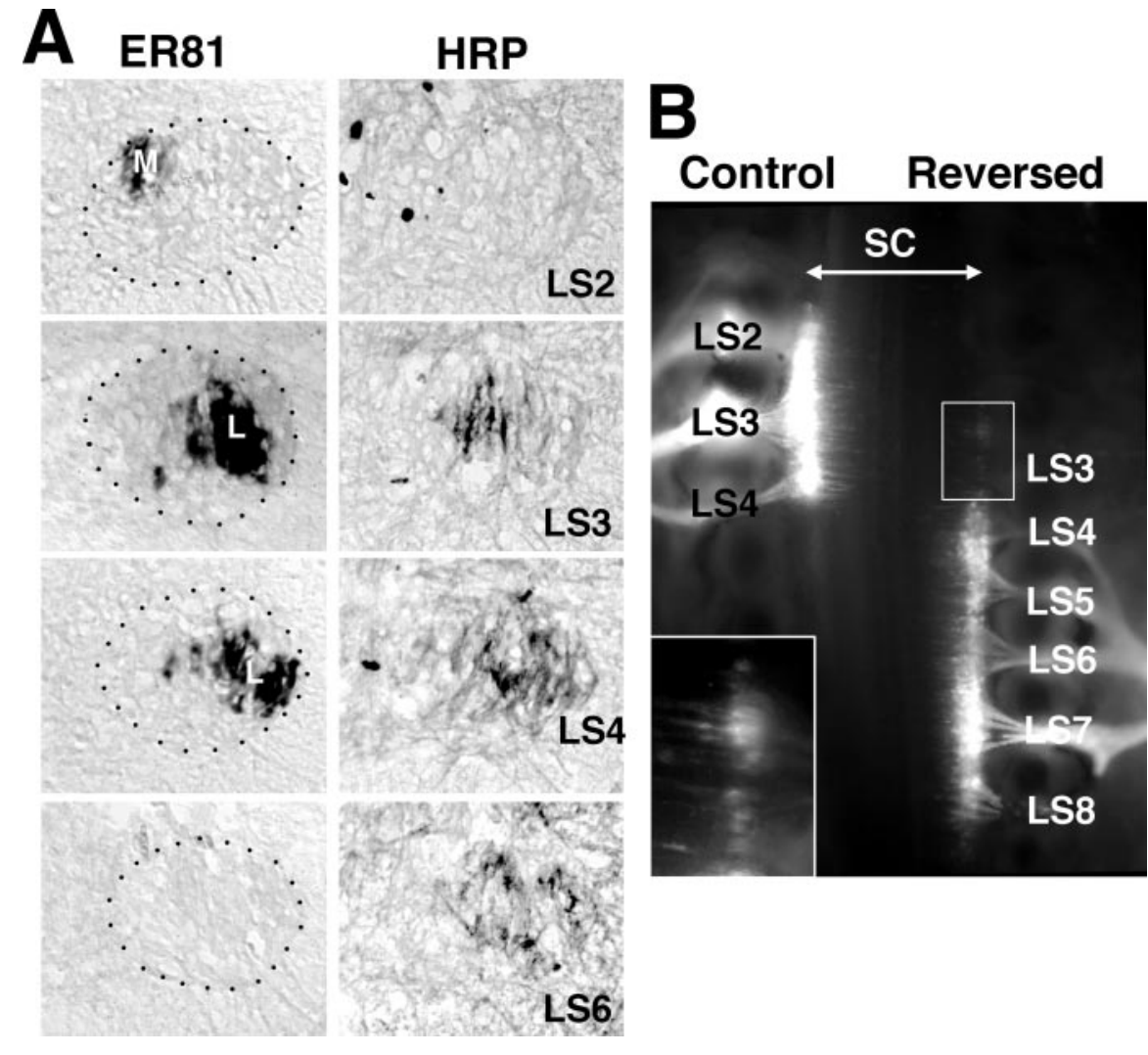

Figure 6. Innervation of the femorotibialis muscle and expression of ER81 are not invariably linked in LLMC motoneurons. $A$, ER81 expression (left panels) and HRP labeling (right panels) in adjacent sections of the LMC innervating an A-P-reversed limb. HRP was injected into the femorotibialis muscle in the reversed limb at St. 32. The LMC in each left panel is outlined by dots; lateral is to the right in each panel. Note that ER81 is expressed in LLMC (L) motoneurons in LS4, one segment more caudal than normal. In contrast, motoneurons in segment LS6 innervated the femorotibialis muscle but did not express ER81. M, ER81-positive MLMC neurons. B, Dil-labeled motoneurons innervating femorotibialis muscles in a St. 32 embryo with an A-P reversed limb (right side); uncleared whole-mount view of the ventral spinal cord (SC). The control femorotibialis muscle is innervated by motoneurons in segments LS2 and LS3, with a small contribution from LS4, whereas the femorotibialis muscle in the reversed limb is innervated by motoneurons in segments LS4 - LS8, with a small contribution from LS3, shown in the inset. Inset is a higher-magnification view of the boxed area. In other embryos with A-P limb reversals $(n=19)$, ER81 was always expressed in LLMC motoneurons in segment LS4 and often in LS5 but never in more caudal motoneurons, although neurons in these caudal segments innervated the femorotibialis muscle, as shown here.

the periphery, whereas Fem (and more caudal) motoneurons were still young enough to respond to peripheral signals.

To test this possibility, we simply removed limb buds from four embryos at St. 16-17, the stage when most other operations were performed, and assayed ER81 expression at St. 28-29, before extensive cell death. As reported previously [Lin et al. (1998), their Fig. $8 H$ ], ER81 expression was markedly reduced in motoneurons on the ablated side. Nevertheless, ER81 was expressed in some Add motoneurons in all embryos but rarely in Fem motoneurons. Moreover, ER81 was expressed in some Add motoneurons when prospective hindlimb tissue was removed even earlier, at St. $15(n=3)$ (Fig. 8). Thus, at the time of our experimental perturbations (St. 16-18), ER81 expression was already established in many Add motoneurons and did not appear to be influenced by mismatched peripheral signals or the subsequent innervation of inappropriate muscles. Interestingly, ER81 expression was not observed in the most rostral Add motoneurons after limb removal at St. 15, suggesting that caudal Add motoneurons may recruit more rostral neurons to the ER81-positive pool, as occurs for Pea3 (Helmbacher et al., 2003). Alternatively, signals from thoracic paraxial mesoderm may inhibit expression in rostral segments when not counterbalanced by inducing signals from adjacent LS mesoderm (cf. Omelchenko and Lance-Jones, 2003). It remains to be determined whether at earlier stages, ER81 expression in MLMC neurons is as malleable as that of LLMC neurons. The finding that the ER81 expression domain extended somewhat caudally in the MLMC in two-thirds of embryos with A-P limb reversals supports this possibility.

\section{Discussion}

The transcription factor ER81 is expressed in two pools of motoneurons, the Add and the external Fem, at lumbosacral levels in the chick. Here we show that ER81 expression and acquisition of target identity are not invariably linked in motoneurons. Competence to express ER81 extends to motoneurons outside the Add and Fem pools, and the pattern of ER81 expression can be influenced by signals from the periphery at stages after the target identity of motoneurons is fixed. We also show that ER81 expression is regulated differently in sensory neurons and motoneurons.

\section{Acquisition of ER81 expression is a multistep process}

The molecular mechanisms that regulate ER81 expression are likely to be complex (cf. Haase et al., 2002; Helmbacher et al., 2003). Any model (supplemental material, available at www.jneurosci.org) of the spatiotemporal distribution and action of the requisite signals must take into account the following observations: (1) ER81 was expressed appropriately in many MLMC motoneurons regardless of the muscle they innervated. (2) ER81 expression in LLMC neurons was more malleable than that in the MLMC. (3) Rostral limb shifts led to attenuation of ER81 in LLMC motoneurons. (4) A-P limb reversal and heterotopic caudal-torostral neural tube transplantation led to an impressive, but spatially restricted, expansion of ER81 expression in LLMC motoneurons. Together, these observations suggest that multiple signals determine the expression pattern of ER81, with the mature pattern sculpted from broader competence domains.

\section{Establishment of competence domains}

Acquisition of motoneuron identity occurs in stages, with different features of motoneurons being programmed at different times (Jessell, 2000; Dasen et al., 2003). Competence to express ER81 represents one aspect of MLMC and LLMC motoneuron identity. In light of the early acquisition of this competence, we expect the signals that determine motoneuron identity and motor pool organization (Liu et al., 2001; Dasen et al., 2003; Omelchenko and Lance-Jones, 2003) also establish ER81 competence domains in MLMC and LLMC neurons. Surprisingly, these competence domains are broader than are normally manifest.

Peripheral signals sculpt ER81 expression patterns

Initiation of ER81 expression requires additional signals from the developing limb (Lin et al., 1998). We show here that these signals 
do not simply activate a preestablished pattern but instead actually shape the pattern, especially in the later-born LLMC motoneurons. Our results suggest the presence of spatially restricted signals in developing limb. They cannot, however, be explained solely by locally distributed cues that are encountered when axons enter limb mesoderm, because some MLMC motoneurons express ER81 after limb bud removal at St. 15-17 [see also Lin et al. (1998), their Fig. $8 \mathrm{H}$ ] when few, if any, axons have extended (Tosney and Landmesser, 1985). It is unlikely that ER81 expression in MLMC motoneurons resulted from residual limb mesoderm in these operated embryos, because LLMC motoneurons in the same embryos did not express ER81. Moreover, ER81 expression in MLMC motoneurons is almost normal after A-P limb reversals in which many axons from segments LS1 and LS2 project to postaxial muscles and apparently never encounter their usual peripheral cues.

Thus, it appears that a diffusible signal, possibly from paraxial mesoderm or somites (cf. Ensini et al., 1998; Sela-Donenfeld and Kalcheim, 2000), can activate ER81 expression, at least in MLMC motoneurons. Our findings suggest that many Add motoneurons in MLMC (some of the earliest-born motoneurons at LS levels) have received sufficient exposure to this signal to initiate and maintain expression of ER81 before their axons have grown to the limb bud. Presumably, motoneurons in the LLMC were more severely affected by experimental manipulations because they have had less exposure to this signal before our experimental surgery, either because they are born later or because the signal is expressed in a steep rostrocaudal gradient. Alternatively, there may be inherent differences in the competence of MLMC and LLMC neurons to respond to this signal.

Our results can also not be explained by a simple A-P gradient of signaling molecules in limb mesoderm, because both limb shifts and A-P limb reversal juxtapose nascent motoneurons to more posterior limb tissue than normal but have dramatically different effects (supplemental material, available at www. jneurosci.org). Instead, our results suggest that motoneurons are exposed to additional specific, locally distributed signals when their axons grow out, pause in the plexus region (Tosney and Landmesser, 1985; Wang and Scott, 2000), and eventually invade the limb.

Although we were unable to assess connectivity and ER81 expression in individual motoneurons, our results for populations of neurons suggest that ER81 is expressed in LLMC motoneurons
LS4-6 $\rightarrow$ LS1-3

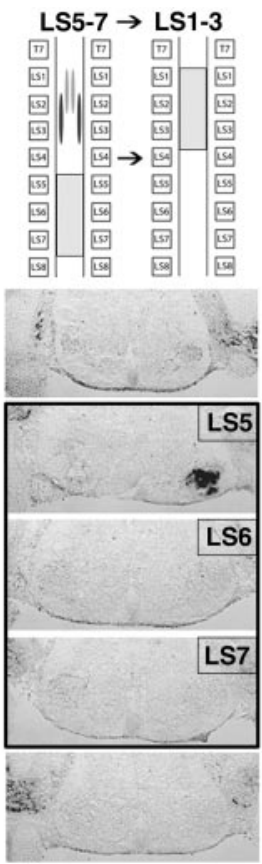



Figure 7. ER81 expression in motoneurons after heterotopic neural tube transplantation at stages after motor pool identity is established. The diagrams at the top of each column depict the operation that was done in the embryo shown in the images directly below. In each diagram, the

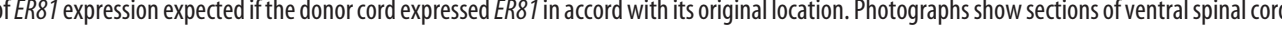

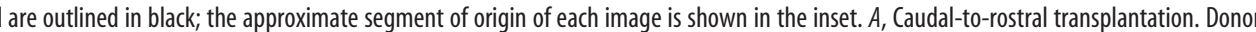
81 was expressed in the LIMC of donor neural tube taken from segments IS3-5 but not in neurons derived from more caudal tissue. peripheral signals. $B$, Rostral-to-caudal transplantation. Note that $E R 81$ was expressed in MLMC neurons in donor tissue transplanted to caudal segments (LS4) in which ER81 is normally never expressed $(n=5)$, suggesting that expression was already fixed in rostral motoneurons at the time of transplantation. 
only when they project preaxially and innervate the Fem muscle. After limb shifts, many motoneurons in segments LS2 and LS3 project postaxially and innervate muscles in posterior limb, thereby accounting for the reduced expression of ER81 in the LLMC in these segments. In contrast, after limb reversal, many motoneurons in LS3 and more caudal segments project to preaxial limb innervate the Fem muscle and express ER81. In only two of the eight embryos in which we assessed both connectivity and ER81 expression was ER81 expressed in rostral LS motoneurons that did not appear to innervate the Fem muscle. There are at least three possible explanations for this apparent discrepancy. First, embryos used for limb reversal were the oldest in this study, so ER81 may have already been established in rostral LLMC motoneurons at the time of surgery. Second, we may occasionally have failed to detect innervation of this large muscle because we recorded e.m.g.s at a single point. Third, as with MLMC motoneurons, target tissue may not provide the direct signal that activates ER81 expression in LLMC motoneurons. However, given the strong correlation of innervation of the Fem muscle and ER81 expression, we believe that entry of axons into preaxial mesoderm, and eventual innervation of the appropriate target muscle, provides a major signal for activating ER81 expression in LLMC motoneurons.

Competence to express ER81 is not always realized

Clearly, projection to preaxial mesoderm and innervation of the Fem muscle are not sufficient to induce expression of ER81 in all segments, because motoneurons in LS6-LS8 that innervated the Fem muscle never expressed ER81. To express ER81, motoneurons must have an inherent competence as well as receive the appropriate peripheral signals. During normal development, motoneurons in segments LS4-LS5 never express ER81, although they are competent to do so. A simple explanation for this observation is that axons of these motoneurons invariably project postaxially and thus are never exposed to the requisite peripheral signals (supplemental material, available at www.jneurosci.org). Because the location of the preaxial-postaxial border varies among embryos, the broad ER81 competence domain of LLMC motoneurons may allow these neurons the flexibility to adopt the correct molecular signature for their ultimate peripheral projection.

Additionally, competent motoneurons in LS4-LS5 may fail to express ER81 as a result of inhibitory signals derived from postaxial mesoderm. After A-P limb reversals, motoneurons in rostral LS segments would be exposed to this inhibitory signal, which could contribute to the observed caudal shift in ER81 expression in LLMC motoneurons. Again, MLMC motoneurons appear to be less severely affected by the proposed inhibitory signals, presumably because their fate is already determined at the time of experimental manipulations.

\section{Regulation of ER81 in sensory neurons and thoracic motoneurons}

Previous studies have shown that ER81 expression is regulated differently in sensory neurons and motoneurons. Sensory neurons require muscle-derived NT3 to express ER81, whereas motoneurons do not (Patel et al., 2003). Our results reinforce and expand these observations by showing that ER81 expression is affected differently in sensory neurons and motoneurons by experimental manipulations. For example, sensory neurons in thoracic DRGs expressed ER81 after limb shifts, whereas thoracic motoneurons did not, even when they innervated the Add muscle.
Thus, thoracic motoneurons, like caudal LMC motoneurons, are not competent to express ER81. Similarly, thoracic motoneurons do not respond to the Pea3-inducing signal GDNF (Haase et al., 2002). This is not surprising, because thoracic spinal cord contains only medial motor column, but not LMC, motoneurons. Together, these findings are consistent with previous studies showing that the phenotype and target specificity of sensory neurons are more plastic than motoneurons (McMahon and Gibson, 1987; Wenner and Frank, 1995; Wang and Scott, 1999, 2002) and that innervation of limb muscles does not convert thoracic motoneurons to LMC neurons (O'Brien and Oppenheim, 1990; Turney et al., 2003).

\section{Summary and conclusions}

In summary, we have shown that acquisition of ER81 expression is established in stages. Initially, early broad competence domains for ER81 expression are established in both MLMC and LLMC neurons in the neural tube. Subsequently, additional signals from the periphery restrict the final pattern of expression in competent neurons to the Add and Fem pools. Expression of other transcription factors in different motor pools most likely follows similar rules. A key challenge for the future is to identify the molecular signals responsible for each stage.

\section{References}

Arber S, Ladle DR, Lin JH, Frank E, Jessell TM (2000) ETS gene Er81 controls the formation of functional connections between group Ia sensory afferents and motor neurons. Cell 101:485-498.

Birren SJ, Lo L, Anderson DJ (1993) Sympathetic neuroblasts undergo a developmental switch in trophic dependence. Development 119:597-610.

Calderó J, Prevette D, Mei X, Oakley RA, Li L, Milligan C, Houenou L, Burek M, Oppenheim RW (1998) Peripheral target regulation of the development and survival of spinal sensory and motor neurons in the chick embryo. J Neurosci 18:356-370.

Dasen JS, Liu JP, Jessell TM (2003) Motor neuron columnar fate imposed by sequential phases of Hox-c activity. Nature 425:926-933.

Ensini M, Tsuchida TN, Belting H, Jessell TM (1998) The control of rostrocaudal pattern in the developing spinal cord: specification of motor neuron subtype identity is initiated by signals from paraxial mesoderm. Development 125:969-982.

Haase G, Dessaud E, Garcès A, de Bovis B, Birling M, Filippi P, Schmalbruch H, Arber S, deLapeyrière O (2002) GDNF acts through PEA3 to regulate cell body positioning and muscle innervation of specific motor neuron pools. Neuron 35:893-905.

Hamburger V, Hamilton HL (1951) A series of normal stages in the development of the chick embryo. J Morphol 88:49-92.

Hamburger V, Levi-Montalcini R (1949) Proliferation, differentiation and degeneration in the spinal ganglia of the chick embryo under normal and experimental conditions. J Exp Zool 111:457-502.

Helmbacher F, Dessaud E, Arber S, deLapeyriere O, Henderson CE, Klein R, Maina F (2003) Met signaling is required for recruitment of motor neurons to PEA3-positive motor pools. Neuron 39:767-777.

Jessell TM (2000) Neuronal specification in the spinal cord: inductive signals and transcriptional codes. Nat Rev Genet 1:20-29.

Lance-Jones C, Landmesser L (1980) Motoneurone projection patterns in embryonic chick limbs following partial deletions of the spinal cord. J Physiol (Lond) 302:559-580.

Lance-Jones C, Landmesser L (1981) Pathway selection by embryonic chick motoneurons in an experimentally altered environment. Proc R Soc Lond B Biol Sci 214:19-52.

Landmesser LT (2001) The acquisition of motoneuron subtype identity and motor circuit formation. Int J Dev Neurosci 19:175-182.

Landmesser LT, O'Donovan MJ (1984) The activation patterns of embryonic chick motoneurones projecting to inappropriate muscles. J Physiol (Lond) 347:205-224

Lin JH, Saito T, Anderson DJ, Lance-Jones C, Jessell TM, Arber S (1998) Functionally related motor neuron pool and muscle sensory afferent subtypes defined by coordinate ETS gene expression. Cell 95:393-407. 
Liu JP, Laufer E, Jessell TM (2001) Assigning the positional identity of spinal motor neurons. Rostrocaudal patterning of Hox-c expression by FGFs, Gdf11, and retinoids. Neuron 32:997-1012.

Matise MP, Lance-Jones C (1996) A critical period for the specification of motor pools in the chick lumbosacral spinal cord. Development 122:659-669.

McMahon SB, Gibson S (1987) Peptide expression is altered when afferent nerves reinnervate inappropriate tissue. Neurosci Lett 73:9-15.

O’Brien MK, Oppenheim RW (1990) Development and survival of thoracic motoneurons and hindlimb musculature following transplantation of the thoracic neural tube to the lumbar region in the chick embryo: anatomical aspects. J Neurobiol 21:313-340.

Omelchenko N, Lance-Jones C (2003) Programming neural Hoxd10: in vivo evidence that early node-associated signals predominate over paraxial mesoderm signals at posterior spinal levels. Dev Biol 261:99-115.

Patel TD, Kramer I, Kucera J, Niederkofler V, Jessell TM, Arber S, Snider WD (2003) Peripheral NT3 signaling is required for ETS protein expression and central patterning of proprioceptive sensory afferents. Neuron 38:403-416.

Price SR, De Marco Garcia NV, Ranscht B, Jessell TM (2002) Regulation of motor neuron pool sorting by differential expression of type II cadherins. Cell 109:205-216.

Scott SA (1984) The effects of neural crest deletions on the development of sensory innervation patterns in embryonic chick hind limb. J Physiol (Lond) 352:285-304.

Sela-Donenfeld D, Kalcheim C (2000) Inhibition of noggin expression in the dorsal neural tube by somitogenesis: a mechanism for coordinating the timing of neural crest emigration. Development 127:4845-4854.

Tosney KW, Landmesser LT (1985) Development of the major pathways for neurite outgrowth in the chick hindlimb. Dev Biol 109:193-214.

Turney BW, Rowan-Hull AM, Brown JM (2003) The innervation of FGFinduced additional limbs in the chick embryo. J Anat 202:83-92.

Wang G, Scott SA (1999) Independent development of sensory and motor innervation patterns in embryonic chick hindlimbs. Dev Biol 208:324-336

Wang G, Scott SA (2000) The "waiting period" of sensory and motor axons in early chick hindlimb: its role in axon pathfinding and neuronal maturation. J Neurosci 20:5358-5366.

Wang G, Scott SA (2002) The development of "normal" dermatomes and somatotopic maps by "abnormal" populations of cutaneous neurons. Dev Biol 251:424-433.

Wang G, Scott SA (2003) Broad competence to express ER81 revealed by surgical manipulations after motoneuron identity is specified. Soc Neurosci Abstr 29:39.11.

Wenner P, Frank E (1995) Peripheral target specification of synaptic connectivity of muscle spindle sensory neurons with spinal motoneurons. J Neurosci 15:8191-8198. 